\title{
Huntingtin in health and disease
}

\author{
Anne B. Young \\ Neurology Service, Massachusetts General Hospital, Boston, Massachusetts, USA \\ J. Clin. Invest. 111:299-302 (2003). doi:10.1172/JCI200317742.
}

After linkage of the Huntington disease (HD) gene was found in 1983, it took ten years of work by an international group to identify the mutation in the gene interesting transcript 15 (IT15) that causes the disease $(1,2)$. $\mathrm{HD}$ is an autosomal dominant inherited neurodegenerative disease that becomes manifest in midlife and causes progressive motor, psychiatric, and cognitive dysfunction. It is invariably terminal. HD symptoms can begin as early as 2 years or as late as 90 years, although the average age of onset is in the late 30s and early 40s. If a child inherits the gene from his or her father, a phenomenon called anticipation frequently occurs, whereby the child's age of onset is lower than the father's (3).

The IT15 gene is composed of 67 exons and encodes a protein of 3,144 amino acids, called huntingtin (2). Exon 1 contains a CAG trinucleotide repeat that encodes the amino acid glutamine, followed by another repeat that encodes proline. In unaffected individuals, there are 10-34 CAG repeats. In those affected by $\mathrm{HD}$, there are more than 40 repeats. In those with 35-39 repeats, the disease is variably penetrant (3-5). The age of onset of the disease varies inversely with the number of CAG repeats. Individuals with juvenile onset usually have over 55 repeats, and they usually inherit the gene from their father. Men occasionally have expanded repeats in their sperm (6). The expansion is thought to occur via slippage during the DNA replication process.

\section{Wild-type huntingtin}

Huntingtin contains a few domains that suggest particular functions, including WW domains and caspase cleavage sites $(7,8)$, but the function of the protein remains unknown. Huntingtin is expressed in the cytoplasm of most cells in the body. In the brain, expression is found predominantly in neurons (2, 9-11). Within the cell, the protein is associated with the endoplasmic reticulum, the microtubules, and organelles such as the mitochondria and synaptic vesicles (12). The association of huntingtin with these cel-

Address correspondence to: Anne B. Young, Neurology Service, VBK-915, Massachusetts General Hospital, 32 Fruit Street, Boston, Massachusetts 02114, USA. Phone: (617) 726-2385; Fax: (617) 726-2353; E-mail: young@helix.mgh.harvard.edu. Conflict of interest: The author has declared that no conflict of interest exists.

Nonstandard abbreviations used: Huntington disease (HD); $\mathrm{N}$-methyl-D-aspartate (NMDA); neuronal intranuclear inclusion (NII). lular components is loose, since during the process of differential centrifugation huntingtin can be separated from them $(10,12)$. The protein contains several caspase cleavage sites (8). Yeast two-hybrid screening studies have shown that huntingtin binds to a number of proteins, including Hap-1, $\alpha$-adaptin, and several others $(7,13,14)$. Additional studies found that huntingtin binds to GAPDH (15). Huntingtin was also recently shown to associate with PSD95, a protein found at the postsynaptic membrane and involved in anchoring of receptor proteins, particularly the $N$-methyl-D-aspartate (NMDA) receptor (16).

The huntingtin protein is necessary for normal development in mice, as knockouts do not survive beyond day 7-8 of embryogenesis (17-19). Conditional knockouts, in which the gene is turned off during adulthood, develop a neurodegenerative disease (20). Recently, cell models of huntingtin expression revealed that the wildtype protein partially protects cells from noxious stimuli or from mutant huntingtin (21). Huntingtin may also be important for cell survival through mechanisms such as growth factor stimulation, as BDNF rescued cells expressing mutant huntingtin (22). Removal of the caspase sites in the protein is beneficial for cells, and wild-type huntingtin protects against mutantinduced cell death (23).

\section{Mutant huntingtin}

There are no antibodies that can distinguish mutant from wild-type protein, although there are antibodies that bind selectively to the polyglutamine repeat (24, 25). Presumed mutant huntingtin is found not only in the cytoplasm but also in the nucleus, where it forms aggregates (or neuronal intranuclear inclusions [NIIs]) (26-28). Aggregates also develop in neurites. The aggregates are ubiquitinated, although antibodies against huntingtin appear to stain more aggregates than do antibodies against ubiquitin. Western blot analysis of HD brain tissue shows full-length huntingtin protein in the nuclear fraction as well as abundant immunopositive bands at lower molecular weight, suggesting proteolytic products in the nucleus. In contrast, in control brains there was full-length protein in the total homogenate but no nuclear protein and few huntingtin fragments in any fraction (26).

Studies of HD brains show that there are more inclusions in the cortex than in the striatum, and that cortical and striatal neurites contain numerous 
aggregates $(11,27)$. Postmortem studies of HD brains also show differential loss of projection neurons containing enkephalin, adenosine A2a, and dopamine D2 receptors compared with cells containing substance $\mathrm{P}$, dynorphin, and dopamine D1 receptors (29-31). In juvenile HD, both types of striatal projection neurons are equally affected (32). In the cortex, neurons in the deeper layers (layers V and VI), which use the neurotransmitter glutamate, develop nuclear and neurite aggregates (27).

Aggregates in HD were first observed in an electromicroscopy study of in vivo biopsies of HD brains (33). This observation was not pursued at the time, but it was remembered in 1996 when studies of the first HD transgenic mouse (28) (expressing the first exon of human huntingtin driven by the huntingtin promoter) were reported. These mice develop normally until around 5 weeks of age, when they begin to lose weight and to perform less well on the Rotorod test. Both brain weight and body weight diminish subsequently. The animals develop diabetes and tremors and become less active. They are finally moribund and die at around 13 weeks (34). Extensive early studies of the brains of these animals showed no clear neurochemical abnormalities like those seen in postmortem HD brains. Electromicroscopy studies, however, showed intranuclear inclusions. In the transgenic mice, it then became obvious from immunocytochemical studies that these NIIs were positive for the HD protein and for ubiquitin. Furthermore, virtually all neurons in the brains of these so-called R6/2 mice contained NIIs (28). These studies led scientists to revisit the examination of human brains in which NIIs were also found $(11,26)$. The frequency of NIIs in human HD brains was lower than in the transgenic mice, and the aggregates appeared as described above.

The formation of aggregates was subsequently thought to be the sine qua non of HD pathogenesis. Two papers then appeared that suggested that the aggregates were an epiphenomenon, since cell death did not necessarily result from neuronal huntingtin aggregation, yet cell death did arise after the expression of mutant huntingtin $(35,36)$. It appeared that, to be toxic, the mutant protein had to get into the nucleus, since constructs with nuclear-export signals attenuated death resulting from exon 1 overexpression. Furthermore, inhibition of caspases rescued cells from death.

Advocates of the hypothesis that aggregates cause cell death have been studying the phenomenon in various in vitro and cell culture assays $(37,38)$. Polyglutamine peptides are not soluble and need to be tied to other proteins to be studied in solution. Tight aggregates apparently form into polar zippers that are held together by hydrogen bonding (39). Some have also hypothesized that transglutaminases link glutamines to lysines covalently (40). No one has yet purified enough of the $\mathrm{N}$-terminal fragment expressing the polyglutamine stretch to perform crystallization. The purified fulllength protein has not been isolated. Aggregation, however, can be studied by fusing a polyglutamine peptide with a GST protein with a sequence that can be broken with trypsin (41). Once the protein is in solution, the GST protein is cleaved off with trypsin, and then the aggregation process can be followed by filter assays. Aggregation is dependent on the length of the polyglutamine repeat. A transition seems to occur in the range of 39-40 repeats. Peptides with fewer than 39-40 glutamines aggregate less robustly than peptides with more than 40 repeats. Cell culture models also show length-dependent polyglutamine aggregation. Highthroughput screens using in vitro and cell culture assays are now being employed to identify compounds that interfere with the aggregation process.

\section{Transgenic mice}

The first transgenic mouse model of HD is described above (34). Numerous additional models have since been created, and each appears to lend new information about the disease (42-45). The ones that have been studied in most detail are the R6/2 and R6/1 mice (34), the N171 mice (42), the YAC72 mice (44), and the conditional exon 1 transgenics and knockouts $(20,46)$. Both strains of R6 mice exhibit little cell death and neuritic pathology but widespread NIIs. N171 mice show striatal cell death and widespread NIIs. N171 mice develop normally but have the onset of progressive neurological decline at about 4 months. In N171 mice, the transgene is driven by the prion promoter. YAC72 mice, which contain the entire human gene and the human promoter, show variable amounts of hyperactivity at about 1 year and then become less active, displaying striatal pathology and evidence of apoptotic cell death.

Examination of signaling proteins in the brains of R6 animals showed changes consistent with an alteration in gene transcription (47). Genes encoding proteins, such as receptors, that had previously been found to change in HD were expressed at lower levels, in a pattern consistent with the human studies. Microarray analysis of the brains of R6 mice, interrogating over 6,500 mouse genes, revealed changes in expression levels mainly of genes involved in signaling pathways (48). R6 mice have been treated with a variety of agents, including free radical scavengers, glutamate antagonists, creatine, and caspase inhibitors, and these are seen to prolong life by about $10-20 \%(49,50)$. Indeed, even environmental enrichment prolongs life by $20-50 \%$ (51). R6/2 mice have been crossed with dominant negative IL-1-converting enzyme (ICE) knockdowns, and offspring live longer than controls (50). Intraventricular infusions of caspase inhibitors also prolong life, as does minocycline (an ICE inhibitor) (52). Electrophysiological studies of cells in brain slices revealed that, early in the disease, there are enhanced NMDA receptor responses, but eventually cells become resistant to NMDA agonists $(53,54)$.

N171 mice exhibited abnormalities that are similar to those found in R6 mice, but they have not been subjected to the same level of examination (48). YAC mice have been examined pathologically but not neurochemically (44).

Conditional exon 1 transgenic mice show behavioral abnormalities, brain atrophy, NIIs, striatal gliosis, and reduction in dopamine D1 receptors when 
the transgene is expressed, and a reversal of these changes when the transgene is turned off in adulthood (46). Conditional knockouts show a progressive neurodegenerative disorder when the wild-type mouse gene is turned off in adulthood (20).

\section{Other whole-organism models}

Worm, fruit fly, and yeast models of HD have been created primarily by transgene approaches using glutamine-encoding trinucleotide expansions (55-57). These models have allowed the identification of suppressors and enhancers of expanded polyglutamineinduced pathology. Heat shock proteins, for example, appear to repress polyQ-induced pathology (58). In PC12 cells (see below), histone deacetylase inhibitors were found to attenuate polyQ-induced toxicity, suggesting that drugs that attenuate histone deacetylation might have therapeutic potential (57). Studies of such compounds are now underway in several transgenic animals.

\section{Cell culture models}

Several cell models of HD are available. Mouse neurons have been fused with mouse teratoma cells (35), and the resulting hybrids have been stably transfected with various polyQ-containing peptides. Another model is based on stably transfected, temperature-sensitive, immortalized mouse striatal neurons (21). PC12 cells have been stably transfected with various genes encoding polyglutamines $(59,60)$. One study of transfected PC12 cells found that CBP protein was sequestered in aggregates in a polyQ-dependent manner, suggesting that genes controlled by CBP may be predominantly affected in the disease (61). Since CBP has histone acetylase activity, these findings suggested that histone deacetylase inhibitors might be used as treatment (57). Indeed, histone deacetylase inhibitors improve phenotype in transgenic flies (57).

\section{Human studies}

In 1996, the Huntington Study Group was formed (62). It is a multi-institutional organization of sites involved in collaborative studies of individuals with HD. Clinical trials of a variety of putative neuroprotective agents have been carried out. These include trials of the glutamate antagonists lamotrigine, remacemide, and riluzole (63). Riluzole and remacemide improved motor function, but lamotragine had no effect. Vitamin E, a free radical scavenger, was not an effective treatment; neither was idebenone, a booster of energy metabolism. Recently, a two-by-two factorial study of coenzyme Q10 and remacemide showed a $13 \%$ slowing of progression with coenzyme Q10 and an improvement in motor function with remacemide, although the results were not statistically significant $(64,65)$. Studies of riluzole, creatine, and minocycline in HD patients are now underway. As we look toward treating individuals in the presymptomatic or early symptomatic phases of the disease, biomarkers or other characteristics of disease onset and progression need to be defined.

\section{Summary}

HD has received at great deal of attention in the field of neuroscience as a model of neurodegeneration. Because it is relatively common and presymptomatic individuals can be identified, investigators have focused on the disease in the hope of finding therapies that can be given to gene carriers presymptomatically to prevent disease development. The results to date suggest that huntingtin is necessary for developing and sustaining normal brain function. In HD, the protein with the expanded polyQ may not function as effectively as the wild-type protein, and this may put neurons (especially in the striatum) under stress. Striatal neurons then become vulnerable to the abundant glutamatergic input from the cerebral cortex. Excitotoxicity, mitochondrial stress, and free radicals increase, and caspases within the cell are activated. Mutant huntingtin is then cleaved, resulting in polyQ-containing fragments that are susceptible to aggregate formation. The mutant fragments and aggregates recruit transcriptional factors vital to the normal function of neurons. Cells survive in a dysfunctional state for some time, and it appears that turning off the mutant gene can result in reversal of neurodegeneration. As many steps in the process of functional decline and cell death represent potential drug targets, we should eventually find a cure.

\section{Acknowledgments}

This work was supported by US Public Health Service grants NS38106 and AG13617, and the Hereditary Disease Foundation.

1. Gusella, J.F., et al. 1983. A polymorphic DNA marker genetically linked to Huntington's disease. Nature. 306:234-238.

2.1993. A novel gene containing a trinucleotide repeat that is unstable in Huntington's disease chromosomes. The Huntington's Disease Collaborative Research Group. Cell. 72:971-983.

3. Young, A.B. 1998. Huntington's disease and other trinucleotide repeat disorders. In Molecular neurology. J.B. Martin, editor. Scientific American Medicine. New York, New York, USA. 35-54.

4. Rubinsztein, D.C., et al. 1996. Phenotypic characterization of individuals with 30-40 CAG repeats in the Huntington disease (HD) gene reveals HD cases with 36 repeats and apparently normal elderly individuals with 36-39 repeats. Am. J. Hum. Genet. 59:16-22.

5. Snell, R.G., et al. 1993. Relationship between trinucleotide repeat expansion and phenotypic variation in Huntington's disease. Nat. Genet. 4:393-397.

6. MacDonald, M.E., et al. 1993. Gametic but not somatic instability of CAG repeat length in Huntington's disease. J. Med. Genet. 30:982-986.

7. Faber, P.W., et al. 1998. Huntingtin interacts with a family of WW domain proteins. Hum. Mol. Genet. 7:1463-1474.

8. Wellington, C.L., et al. 1998. Caspase cleavage of gene products associated with triplet expansion disorders generates truncated fragments containing the polyglutamine tract. J. Biol. Chem. 273:9158-9167.

9. Landwehrmeyer, G.B., et al. 1995. Huntington's disease gene: regional and cellular expression in brain of normal and affected individuals. Ann. Neurol. 37:218-230.

10. DiFiglia, M., et al. 1995. Huntingtin is a cytoplasmic protein associated with vesicles in human and rat brain neurons. Neuron. 14:1075-1081.

11. Ferrante, R.J., et al. 1997. Heterogeneous topographic and cellular distribution of Huntington expression in the normal human neostriatum. J. Neurosci. 17:3052-3063.

12. Velier, J., et al. 1998. Wild-type and mutant huntingtins function in vesicle trafficking in the secretory and endocytic pathways. Exp. Neurol. 152:34-40.

13. Li, X.J., et al. 1995. A huntingtin-associated protein enriched in brain with implications for pathology. Nature. 378:398-402.

14. Kalchman, M.A., et al. 1997. HIP1, a human homologue of S. cerevisiae Sla2p, interacts with membrane-associated huntingtin in the brain. Nat. Genet. 16:44-53.

15. Burke, J.R., et al. 1996. Huntingtin and DRPLA proteins selectively interact with the enzyme GAPDH. Nat. Med. 2:347-350. 
16. Sun, Y., Savanenin, A., Reddy, P.H., and Liu, Y.F. 2001. Polyglutamineexpanded huntingtin promotes sensitization of N-Methyl-D-aspartate receptors via post-synaptic density 95. J. Biol. Chem. 276:24713-24718.

17. Duyao, M.P., et al. 1995. Inactivation of the mouse Huntington's disease gene homolog Hdh. Science. 269:407-410.

18. O'Kusky, J.R., Nasir, J., Cicchetti, F., Parent, A., and Hayden, M.R. 1999. Neuronal degeneration in the basal ganglia and loss of pallido-subthalamic synapses in mice with targeted disruption of the Huntington's disease gene. Brain Res. 818:468-479.

19. Dragatsis, I., Efstratiadis, A., and Zeitlin, A. 1998. Mouse mutant embryos lacking huntingtin are rescued from lethality by wild-type extraembryonic tissues. Development. 125:1529-1539.

20. Dragatsis, I., Levine, M.S., and Zeitlin, S. 2000. Inactivation of Hdh in the brain and testis results in progressive neurodegeneration and sterility in mice. Nat. Genet. 26:300-306.

21. Rigamonti, D., et al. 2000. Wild-type huntingtin protects from apoptosis upstream of caspase-3. J. Neurosci. 20:3705-3713.

22. Zuccato, C., et al. 2001. Loss of huntingtin-mediated BDNF gene transcription in Huntington's disease. Science. 293:493-498.

23. Leavitt, B.R., et al. 2001. Wild-type huntingtin reduces the cellular toxicity of mutant huntingtin in vivo. Am. J. Hum. Genet. 68:313-324.

24. Persichetti, F., et al. 1996. Differential expression of normal and mutant Huntington's disease gene alleles. Neurobiol. Dis. 3:183-190.

25. Trottier, Y., et al. 1995. Cellular localization of the Huntington's disease protein and discrimination of the normal and mutated form. Nat. Genet. 10:104-110.

26. DiFiglia, M., et al. 1997. Aggregation of huntingtin in neuronal intranuclear inclusions and dystrophic neurites in brain. Science. 277:1990-1993.

27. Sapp, E., et al. 1997. Huntingtin localization in brains of normal and Huntington's disease patients. Ann. Neurol. 42:604-612.

28. Davies, S.W., et al. 1997. Formation of neuronal intranuclear inclusions underlies the neurological dysfunction in mice transgenic for the HD mutation. Cell. 90:537-548.

29. Reiner, A., et al. 1988. Differential loss of striatal projection neurons in Huntington disease. Proc. Natl. Acad. Sci. USA. 85:5733-5737.

30. Albin, R.L., et al. 1990. Abnormalities of striatal projection neurons and $\mathrm{N}$-methyl-D-aspartate receptors in presymptomatic Huntington's disease. N. Engl. J. Med. 322:1293-1298.

31. Glass, M., Dragunow, M., and Faull, R.L. 2000. The pattern of neurodegeneration in Huntington's disease: a comparative study of cannabinoid, dopamine, adenosine and $\mathrm{GABA}(\mathrm{A})$ receptor alterations in the human basal ganglia in Huntington's disease. Neuroscience. 97:505-519.

32. Albin, R.L., Reiner, A., Anderson, K.D., Penney, J.B., and Young, A.B. 1990. Striatal and nigral neuron subpopulations in rigid Huntington's disease: implications for the functional anatomy of chorea and rigidityakinesia. Ann. Neurol. 27:357-365.

33. Roizin, L., Stellar, S., and Liu, J.C. 1979. Neuronal nuclear-cytoplasmic changes in Huntington's chorea: electron microscope investigations. In Advances in neurology. Volume 23. T.N. Chase, N.S. Wexler, and A. Barbeau, editors. Raven Press. New York, New York, USA. 95-122.

34. Mangiarini, L., et al. 1996. Exon 1 of the $H D$ gene with an expanded CAG repeat is sufficient to cause a progressive neurological phenotype in transgenic mice. Cell. 87:493-506.

35. Kim, M., et al. 1999. Mutant huntingtin expression in clonal striatal cells: dissociation of inclusion formation and neuronal survival by caspase inhibition. J. Neurosci. 19:964-973.

36. Saudou, F., Finkbeiner, S., Devys, D., and Greenberg, M.E. 1998. Huntingtin acts in the nucleus to induce apoptosis but death does not correlate with the formation of intranuclear inclusions. Cell. 95:55-66.

37. Scherzinger, E., et al. 1997. Huntingtin-encoded polyglutamine expansions form amyloid-like protein aggregates in vitro and in vivo. Cell. 90:549-558.

38. Huang, C.C., et al. 1998. Amyloid formation by mutant huntingtin: threshold, progressivity and recruitment of normal polyglutamine proteins. Somat. Cell Mol. Genet. 24:217-233.

39. Perutz, M.F., Johnson, T., Suzuki, M., and Finch, J.T. 1994. Glutamine repeats as polar zippers: their possible role in inherited neurodegenerative diseases. Proc. Natl. Acad. Sci. USA. 91:5355-5358.

40. Green, D.R. 1999. Harm's way: polyglutamine repeats and the activation of an apoptotic pathway. Neuron. 22:416-417.

41. Hollenbach, B., et al. 1999. Aggregation of truncated GST-HD exon 1 fusion proteins containing normal range and expanded glutamine repeats. Philos. Trans. R. Soc. Lond. B Biol. Sci. 354:991-994.

42. Schilling, G., et al. 1999. Intranuclear inclusions and neuritic aggregates in transgenic mice expressing a mutant $\mathrm{N}$-terminal fragment of huntingtin. Hum. Mol. Genet. 8:397-407.

43. Reddy, P.H., et al. 1999. Transgenic mice expressing mutated full-length HD cDNA: a paradigm for locomotor changes and selective neuronal loss in Huntington's disease. Philos. Trans. R. Soc. Lond. B Biol. Sci. 354:1035-1045

44. Hodgson, J.G., et al. 1999. A YAC mouse model for Huntington's disease with full-length mutant huntingtin, cytoplasmic toxicity, and selective striatal neurodegeneration. Neuron. 23:181-192.

45. Ordway, J.M., et al. 1997. Ectopically expressed CAG repeats cause intranuclear inclusions and a progressive late onset neurological phenotype in the mouse. Cell. 91:753-763.

46. Yamamoto, A., Lucas, J.J., and Hen, R. 2000. Reversal of neuropathology and motor dysfunction in a conditional model of Huntington's disease. Cell. 101:57-66.

47. Cha, J.-H.J., et al. 1998. Altered brain neurotransmitter receptors in transgenic mice expressing a portion of an abnormal human Huntington disease gene. Proc. Natl. Acad. Sci. USA. 95:6480-6485.

48. Luthi-Carter, R., et al. 2000. Decreased expression of striatal signaling genes in a mouse model of Huntington's disease. Hum. Mol. Genet. 9:1259-1271.

49. Ferrante, R.J., et al. 2000. Neuroprotective effects of creatine in a transgenic mouse model of Huntington's disease. J. Neurosci. 20:4389-4397.

50. Ona, V.O., et al. 1999. Inhibition of caspase-1 slows disease progression in a mouse model of Huntington's disease. Nature. 399:263-267.

51. Carter, R.J., Hunt, M.J., and Morton, A.J. 2000. Environmental stimulation increases survival in mice transgenic for exon 1 of the Huntington's disease gene. Mov. Disord. 15:925-937.

52. Chen, M., et al. 2000. Minocycline inhibits caspase-1 and caspase-3 expression and delays mortality in a transgenic mouse model of Huntington disease. Nat. Med. 6:797-801.

53. Levine, M.S., et al. 1999. Enhanced sensitivity to N-methyl-D-aspartate receptor activation in transgenic and knockin mouse models of Huntington's disease. J. Neurosci. Res. 58:515-532.

54. Hansson, O., et al. 1999. Transgenic mice expressing a Huntington's disease mutation are resistant to quinolinic acid-induced striatal excitotoxicity. Proc. Natl. Acad. Sci. USA. 96:8727-8732.

55. Sipione, S., and Cattaneo, E. 2001. Modeling Huntington's disease in cells, flies, and mice. Mol. Neurobiol. 23:21-51.

56. Faber, P.W., Alter, J.R., MacDonald, M.E., and Hart, A.C. 1999. Polyglutamine-mediated dysfunction and apoptotic death of a Caenorhabditis elegans sensory neuron. Proc. Natl. Acad. Sci. USA. 96:179-184.

57. Steffan, J.S., et al. 2001. Histone deacetylase inhibitors arrest polyglutamine-dependent neurodegeneration in Drosophila. Nature. 413:739-743.

58. Satyal, S.H., et al. 2000. Polyglutamine aggregates alter protein folding homeostasis in Caenorhabditis elegans. Proc. Natl. Acad. Sci. USA. 97:5750-5755.

59. Li, S.H., Cheng, A.L., Li, H., and Li, X.J. 1999. Cellular defects and altered gene expression in PC12 cells stably expressing mutant huntingtin. J. Neurosci. 19:5159-5172.

60. Kazantsev, A., Preisinger, E., Dranovsky, A., Goldgaber, D., and Housman, D. 1999. Insoluble detergent-resistant aggregates form between pathological and nonpathological lengths of polyglutamine in mammalian cells. Proc. Natl. Acad. Sci. USA. 96:11404-11409.

61. Steffan, J.S., et al. 2000. The Huntington's disease protein interacts with p53 and CBP and represses transcription. Proc. Natl. Acad. Sci. USA. 97:6763-6768.

62. Kieburtz, K., et al. 1996. Unified Huntington's disease rating scale: reliability and consistency. Mov. Disord. 11:136-142.

63. Kieburtz, K. 1999. Antiglutamate therapies in Huntington's disease. J. Neural Transm. Suppl. 55:97-102.

64. Schilling, G., Coonfield, M.L., Ross, C.A., and Borchelt, D.R. 2001. Coenzyme Q10 and remacemide hydrochloride ameliorate motor deficits in a Huntington's disease transgenic mouse model. Neurosci. Lett. 315:149-153.

65. 2001. A randomized, placebo-controlled trial of coenzyme Q10 and remacemide in Huntington's disease. Neurology. 57:397-404. 